EPJ Web of Conferences 81, 05029 (2014)

DOI: $10.1051 /$ epjconf/ 20148105029

(C) Owned by the authors, published by EDP Sciences, 2014

\title{
Search for baryonium and the physics at FAIR
}

\author{
S. Wycech ${ }^{1, a}$, J-P. Dedonder ${ }^{2}$, and B. Loiseau ${ }^{2}$ \\ ${ }^{1}$ National Centre for Nuclear Studies, Warsaw, Poland \\ ${ }^{2}$ Sorbonne Universités, Université Pierre et Marie Curie, Sorbonne Paris Cité, Université Paris Diderot, et \\ IN2P3-CNRS, UMR 7585, LPNHE, 4 place Jussieu, 75252 Paris, France
}

\begin{abstract}
The existence of nucleon-antinucleon quasi-bound state is indicated in two decay modes of the $J / \psi$ meson studied by BES. We discuss an explanation in a fairly traditional Paris-potential model of the $N \bar{N}$ interactions. A broad, $S$-wave and a narrow $P$-wave quasi-bound states are predicted by this model. Some existing experimental evidence and possible verifications in the future are indicated.
\end{abstract}

\section{Introduction}

Baryonium understood as a nucleon-antinucleon quasi-bound was searched for at CERN in the days of LEAR. Nothing has been found, but broad states or states close to the threshold were not excluded [1, 2]. One possible reason of the failure is the heavy background due to annihilation processes. Another is the large number of allowed partial vaves. A convincing detection requires selective experiments, and the first such experiment was performed by the BES Collaboration [3] who studied the decay

$$
J / \psi \rightarrow \gamma p \bar{p}
$$

The selectivity in this reaction is due to the definite initial state and CP invariance. As shown in figure 3 the measured spectrum of $p \bar{p}$ invariant mass displays two prominent maxima named initially $X(1859)$ and $X(2170)$. The first one, close to the threshold, is apparently due to strong attraction in the final iso-singlet spin-singlet ${ }^{11} S_{0}$ wave which is one of three partial waves allowed in reaction (1). Indeed, calculations of final state interactions (FSI) performed with Paris [4] and Jülich [5] potential indicate a strong threshold enhancement. The basic difference is that with the Paris potential the threshold peak is due to a bound state and with the Jülich potential it results from a virtual state. To distinguish between these two possibilities, one needs to look directly below the $p \bar{p}$ threshold. Several methods to do this are discussed in section 2.

\subsection{Final state interactions in $J / \psi$ decays}

In this note we report an extension of the FSI calculations of Ref. [4] which now covers the whole photon spectrum. The basic assumption of this approach (also that in Ref. [5]) is that the photon is emitted before the baryons are formed. The two related processes are plotted in diagrams 1,2 and

ae-mail: wycech@fuw.edu.pl 
the FSI is calculated in terms of half-off shell $T$ matrix generated by the Paris potential [6]. This approach allows to calculate the spectrum but not the absolute decay rate. One free parameter, the radius $R(=0.28 \mathrm{fm})$ of a Gaussian source function is used to describe the creation of a $\gamma p \bar{p}$ state (see figure 1). However, in order to reproduce in a better way both maxima $X(1859)$ and $X(2170)$, it turns out profitable to assume the radius to be weakly dependent on the photon energy. It was found to change from $0.28 \mathrm{fm}$ at maximal $k \sim 1.2 \mathrm{GeV}$ to $0.39 \mathrm{fm}$ at $k=0$.
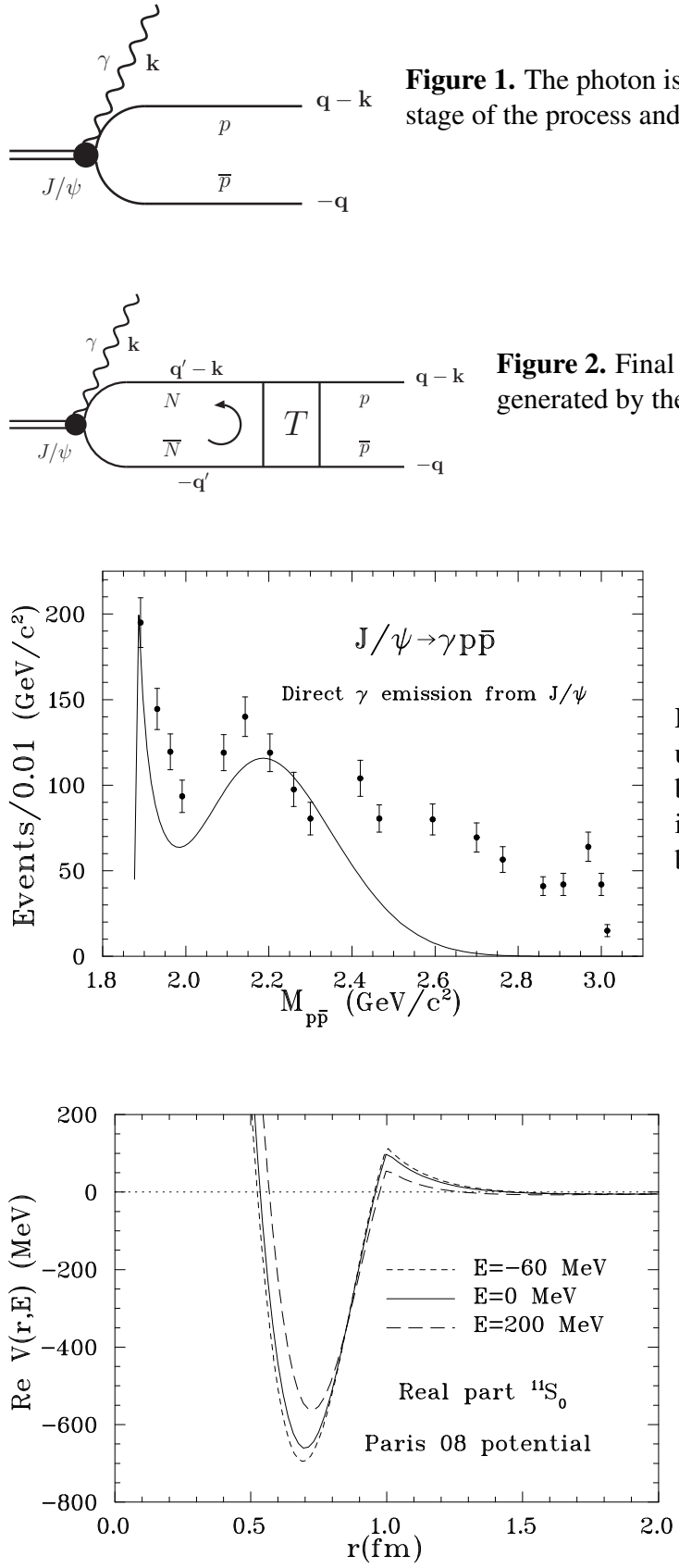

Figure 1. The photon is emitted either from $J / \psi$ or during the hadronisation stage of the process and the final baryons are formed in the $S$ wave

Figure 2. Final state interactions described by half-off shell $T$ matrix generated by the Paris potential
Figure 3. The $p \bar{p}$ invariant mass spectrum obtained under the assumption that photon is emitted before the baryons are formed. The missing strength at large $p \bar{p}$ invariant mass, $M_{\bar{p}}$, comes from the photon radiated by final hadrons [7]

Figure 4. The Paris $N \bar{N}$ real potential in the ${ }^{11} S_{0}$ wave. It generates a $50 \mathrm{MeV}$ broad quasi-bound state at $\sim 5 \mathrm{MeV}$ binding. The well and barrier structure generate the shape resonance visible in the spectrum in figure 3 at $2170 \mathrm{MeV}$ 
Inspection of figure 3 shows that both states may be reproduced by the potential $N \bar{N}$ interactions related at large distances via G-parity transformation to the $N N$ interactions. However, the proper description of both peaks involves distant extrapolation of the $T_{N \bar{N}}$ matrices off energy shell, which corresponds to very short ranged interactions. This figure shows also that a sizable portion of the spectrum is missing. The missing part comes from photon emissions by final baryons: $N \bar{N}$ and exchange currents [7]. Similar final state emission model offers a consistent description of the rate and the spectra in cases of $\pi, \omega, \phi$ mesons with a constant $R=.28 \mathrm{fm}$ radius. This knowledge of initial formation radius allows to calculate inverse reactions leading to formation of $J / \psi$ and a meson in nuclear collisions of $\bar{p}$ [7].

\section{Looking below the $N \bar{N}$ threshold}

Testing the subthreshold amplitudes may be realised in few body systems in particular in light antiprotonic atoms or at extreme nuclear peripheries. In these conditions nucleons are bound and the effective subthreshold energies are composed of binding energies and recoil of the $N \bar{p}$ pair with respect to the rest of the system. For valence nucleons the $-E_{\text {binding }}-E_{\text {recoil }}$ may reach down to $-40 \mathrm{MeV}$. Here, we want to point out one experiment that might be of interest at FAIR/FLAIR. Table 1 shows the ratios of antiproton capture rates on neutrons and protons $C(n \bar{p}) / C(p \bar{p})$ bound to nuclear peripheries. These reflect the ratios of neutron and proton densities. The second and third columns indicate such ratios extracted from widths of two antiprotonic atomic levels the "lower" and the "upper" one. These widths are determined at nuclear densities $\sim 10 \%$ and $\sim 5 \%$ of the central density $\rho_{0}$. The last column is obtained with radiochemical studies of final nuclei with one neutron or one proton removed in the annihilation reaction [8]. The latter process is localised at densities $\rho \sim 10^{-3} \rho_{0}$. In standard nuclei shown in the upper part of the table the ratios $C(n \bar{p}) / C(p \bar{p}) \sim \rho_{n} / \rho_{p}$ increase at nuclear peripheries. However, in some nuclei characterised by a small proton binding, indicated in the lower part of the table, the ratio $C(n \bar{p}) / C(p \bar{p})$ suddenly drops at extreme nuclear peripheries. That effect cannot be explained by the nuclear structure alone and we attribute it to the existence of a narrow bound state in the $N \bar{N}$ system. Such a narrow state is in fact predicted by the Paris potential in the ${ }^{33} P_{1}$ wave, see table 2 .

Table 1. Ratios of $N(n \bar{p})$ and $N(p \bar{p})$ capture rates from atomic states. The last column shows experimental numbers from radiochemical experiments. Other columns (see text) give ratios calculated with optical potential and plausible nuclear densities based on experimental results from Ref. [8].

\begin{tabular}{llll}
\hline atom & lower & upper & radiochemistry \\
\hline${ }^{96} \mathrm{Zr}$ & $0.95(9)$ & $1.53(29)$ & $2.6(3)$ \\
${ }^{124} \mathrm{Sn}$ & $1.79(10)$ & $2.44(39)$ & $5.0(6)$ \\
\hline${ }^{106} \mathrm{Cd}$ & $1.64(80)$ & $2.10(80)$ & $0.5(1)$ \\
${ }^{112} \mathrm{Sn}$ & $1.90(13)$ & $2.43(49)$ & $0.79(14)$
\end{tabular}

Another way to look below threshold is opened by the BES collaboration experiment [9] detecting radiative $J / \psi$ decays

$$
J / \psi \rightarrow \gamma \pi^{+} \pi^{-} \eta^{\prime}
$$

and observation that the mesons are correlated to another state named $X(1835)$ with quantum numbers consistent with the $X(1859)$ state. Within the Paris-potential interpretation, the $X(1835)$ is due to the interference of the baryonium ${ }^{11} S_{0}$ state, seen above the threshold, with a background formation amplitude [10]. 
Table 2. Binding energies in $\mathrm{MeV}$ of the close to threshold quasi-bound states in the Paris potential [6]

\begin{tabular}{ll}
\hline${ }^{2 T+1}{ }^{2 S+1} L_{J}$ & $E-i \Gamma / 2$ \\
\hline${ }^{11} S_{0}$ & $-4.8-\mathrm{i} 26$ \\
${ }^{33} P_{1}$ & $-4.5-19.0$ \\
\hline
\end{tabular}

\subsection{Experiments suggested}

Following the indications from BES experiments the baryonia should be searched in the region of $0-60 \mathrm{MeV}$ below the $N \bar{N}$ threshold. It would be advisable to repeat two old experiments at different energies, possibly with polarized particles:

- Search for narrow signals in the $\gamma$-spectrum from $p \bar{p}$ annihilation was performed at rest [2], The signals (in the region that we expect them now to exist) were covered by heavy background due to $\pi^{0}$ decays and $\pi^{-} p \rightarrow \gamma n$. It would be better to perform this experiment with few hundred $\mathrm{MeV}$ antiprotons which would shift the expected signal away from the region of high background.

- The $\bar{p} d \rightarrow n X$ experiment [1] was performed at $1.3 \mathrm{GeV} / \mathrm{c}$. This gives rather small chance of $p \bar{p}$ coupling in the statistically insignificant ${ }^{11} S_{0}$ wave. Lower energies would be better.

New instructive experiments that possibly could be performed in the coming years are:

- Fine structure splitting in light antiprotonic atoms ${ }^{1} \mathrm{H},{ }^{2} \mathrm{H},{ }^{3} \mathrm{H},{ }^{3} \mathrm{He},{ }^{4} \mathrm{He}$ would allow to trace energy dependence of the selected $\bar{p} N$ amplitudes in the subthreshold region down to $\sim-40 \mathrm{MeV}$.

- Studies of mesons emitted from annihilations of $\bar{p}$ at nuclear peripheries. In particular nuclei with closed shells with one loosely bound valence nucleon could be profitable. In the latter case the baryonium signal would be separated from a complicated background due to other annihilation channels.

\section{Acknowledgements}

This work has been partially supported by a grant from the French-Polish exchange program COPIN/CNRSIN2P3, collaboration 05-115. SW was also supported by Narodowe Centrum Nauki grant 2011/03/B/ST2/00270.

\section{References}

[1] R. Bertini et al., Nucl. Phys. B 209, 269 (1982).

[2] L. Adiels et al., Phys. Lett. B 182, 405 (1986).

[3] J. Z. Bai et al., (BES Collaboration) Phys. Rev. Lett. 91, 022001 (2003) and M. Ablikim et al., (BES Collaboration), Phys. Rev. Lett. 108, 112003 (2012).

[4] B. Loiseau and S. Wycech, Phys. Rev. C 72, 011001 (2005).

[5] J. Haidenbauer, Ulf-G. Meißner and A. Sibirtsev, Phys. Rev. D 74, 017501 (2006).

[6] B. El-Bennich, M. Lacombe, B. Loiseau and S.Wycech, Phys. Rev. C 79, 054001 (2009).

[7] J-P. Dedonder, B. Loiseau and S. Wycech, to be published.

[8] P. Lubinski et al., Phys. Rev. C 57, 2962 (1997).

[9] M. Ablikim et al., (BES Collaboration), Phys. Rev. Lett. 95, 262001 (2005)

[10] J-P. Dedonder, B. Loiseau, B. El-Bennich and S. Wycech, Phys. Rev. C 80, 045207(2009). 